\title{
Reduction of the Weaving Process Set-up Time through Multi-Objective Self- Optimization
}

\author{
Saggiomo $\mathbf{M}^{*}$, Gloy $\mathrm{YS}$ and Gries T
}

Institut für Textiltechnik (ITA) der RWTH Aachen University, Aachen, Germany

\begin{abstract}
Real (physical) objects melt together with information-processing (virtual) objects. These blends are called Cyber-Physical Production Systems (CPPS). The German government identifies this technological revolution as the fourth step of industrialization (Industry 4.0). Through embedding of intelligent, self-optimizing CPPS in process chains, productivity of manufacturing companies and quality of goods can be increased. Textile producers especially in high-wage countries have to cope with the trend towards smaller lot sizes in combination with the demand for increasing product variations. One possibility to cope with these changing market trends consists in manufacturing with CPPS and cognitive machinery. This paper focuses on woven fabric production and presents a method for multiobjective self-optimization of the weaving process. Multi-objective self-optimization assists the operator in setting weaving machine parameters according to the objective functions warp tension, energy consumption and fabric quality. Individual preferences of customers and plant management are integrated into the optimization routine. The implementation of desirability functions together with Nelder/Mead algorithm in a software-based Programmable Logic Controller (soft-PLC) is presented. The self-optimization routine enables a weaving machine to calculate the optimal parameter settings autonomously. Set-up time is reduced by $75 \%$ and objective functions are improved by at least $14 \%$ compared to manual machine settings.
\end{abstract}

Keywords: Weaving; Industry 4.0; Optimization; Cognition; Set-up time

\section{Introduction}

Weaving is the most common as well as the oldest process for fabric manufacturing. Until today a fabric is created by crossing warp and weft threads in a right angle, like it was done since approximately 4000 B.C. Today's applications are for example:

- Apparel (jeans, lining fabric, etc.),

- Geotextiles (erosion protection, soil reinforcement, etc.) and

- Technical textiles (filters, fireproof fabric, airbag fabric, reinforcements for fiber composites, etc.).

Because of the low production costs, the textile production has been relocated to the Asian countries, whereas the production of high-quality and technical textiles is progressively shifted to Europe. The textile industry in high-wage countries like Germany is facing numerous challenges today. For example, the tendency to small lot sizes requires shorter cycle times and aggravates the economical production of goods $[1,2]$.

Small lot sizes in the fabric production often involve a change of the fabric. A weaving machine with about 200 parameters has to be reconfigured after each change of the fabric to fulfill the expectations of the customer. In order to find the optimal configuration for the machine, the operator of the weaving machine has to conduct weaving trials. These time-consuming and wasteful trials require - depending on the experience of the operator - the weaving of up to $120 \mathrm{~m}$ of fabric until the optimal parameters are found $[3,4]$. This research paper presents an algorithm for multi-objective self-optimization of the weaving process and the integration of this algorithm into the machine control of the weaving machine. A weaving machine is upgraded to a cognitive unit (CPPS) on the shop floor. The algorithm for self-optimization identifies a combination of machine parameters, with the result that the essential objective functions can be adjusted to the individual preferences. Figure 1 visualizes the principle of a weaving machine.

\section{Concept and Implementation of Multi-Objective Self- Optimization of the Weaving Process}

Self-optimization systems apply adoptions of their inner state or structure in case of changes in input conditions or disturbances. Target values for self-optimization can be e.g. capacity, lot size, quality or energy consumption [1]. According to [5] self-optimization systems are characterized by the following continuous steps:

- Analysis of actual situation.

- Determination of targets.

- Adaption of system behaviour in order to reach the targets.

The presented concept of self-optimizing production systems will now be applied to the weaving process. The following objective functions are considered by the multi-objective self-optimization (MOSO) of the weaving process:

- Warp tension,

- Energy consumption of the weaving machine (air- and active power consumption),

- Quality of the fabric,

The objective functions are optimized according to the following parameters:

*Corresponding author: Marco Saggiomo, Institut für Textiltechnik der RWTH Aachen University, Aachen, Germany, Tel: 49-241-803470; Fax: 49-241-8022422; E-mail: Marco.Saggiomo@ita.rwth-aachen.de

Received November 20, 2015; Accepted June 06, 2016; Published June 13, 2016

Citation: Saggiomo M, Gloy YS, Gries T (2016) Reduction of the Weaving Process Set-up Time through Multi-Objective Self-Optimization. J Textile Sci Eng 6: 255. doi:10.4172/2165-8064.1000255

Copyright: (c) 2016 Saggiomo M, et al. This is an open-access article distributed under the terms of the Creative Commons Attribution License, which permits unrestricted use, distribution, and reproduction in any medium, provided the original author and source are credited. 


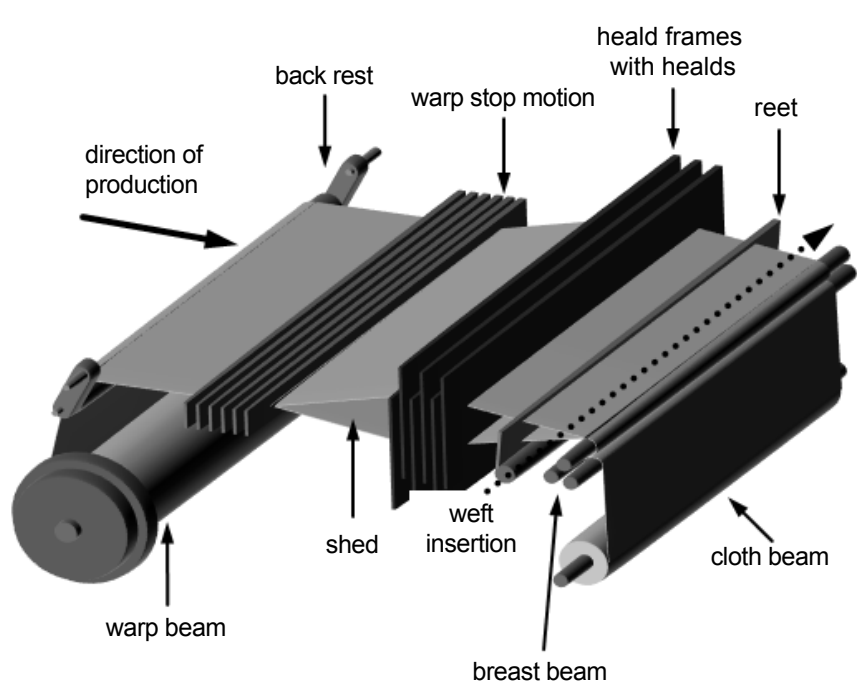

Figure 1: Setup of a weaving machine [4].

- Basic warp tension (bwt),

- Revolutions per minute (n),

- Vertical warp stop motion position $\left(\right.$ wsm $\left._{\mathrm{y}}\right)$,

With the MOSO of the weaving process, a weaving machine is enabled to automatically find an optimal configuration. A program for self-optimization is implemented in a programmable logic controller (PLC). Figure 2 provides an overview of the required hard- and software infrastructure.

\section{Signal processing and control system}

For the signal processing and the execution of the self-optimisation routine the ibaPADU-S Module system by iba AG, Fürth, Germany is used. The System consists of the following modules:

- ibaMS16xAI-20 mA: Analog input module for current signals in the range of $(0 \ldots 20) \mathrm{mA}$

- ibaMS16xAl-10 V: Analog input module for voltage signals in the range of $(-10 \ldots 10) \mathrm{V}$

- ibaPADU-S-IT-16: Central Processing Unit (CPU) for the modular system

The analog input modules ibaMS16xAI-20 mA and ibaMS16xAl-10 $\mathrm{V}$ collect and process the signals from the sensor system. Both analog input modules are connected to the base unit ibaPADU-S-IT-16 using a back panel bus. The base unit receives the data from the analog input modules through the back panel bus.

The central unit ibaPADU-S-IT-16 is connected to a computer using the Transmission Control Protocol/Internet Protocol (TCP/IP) interface. On the computer the software ibaLogicV4 from iba AG is installed. IbaLogicV4 is a programming environment which forms a software-based programmable logic controller (soft-PLC) together with the introduced modular system. An ibaLogicV4 program is created on the computer and transmitted to the central unit using TCP/ IP. The central unit provides a runtime platform for the ibaLogicV4 program (runtime system). In the ibaLogicV4 program the data from the analog input modules are collected and processed. The program for $\mathrm{MOSO}$ is developed within the environment of ibaLogicV4 and uses the ibaPADU-S-IT-16 module as runtime system. The program for MOSO is presented in section II.D.

\section{Measurement technology for warp tension}

For measuring the warp tension, the yarn tension sensor TS44/ A250 by BTSR International S.p.A. Partita, Olgiate Olona, Italy is used. The yarn tension sensor generates a voltage signal in the range of $(0 \ldots 10) \mathrm{V}$ which is proportional to the present yarn tension. The yarn tension sensor is placed in the middle of the weaving machine, between the back rest and the warp stop motion. The data connector of the yarn tension sensor is connected to the soft-PLC using an analog/digital converter. For additional information on the yarn tension sensor.

\section{Measurement technology for energy consumption}

Air consumption measurement: The air consumption of the weaving machine is measured using the flow sensor SD8000 by ifm Electronic GmbH, Essen, Germany. The flow sensor generates a signal, which is proportional to the compressed air consumption in the range of $(4 \ldots 20) \mathrm{mA}$. The output data from the flow senor are wirelessly transferred to the soft-PLC.

Active power measurement: The power measurement module collects characteristic values of the three-phase supply and saves the values into the process image. To access the measurement values from the power measurement module, the power measurement module is connected to a Fieldbus controller using a terminal bus.

The process image of the power measurement module is provided to the fieldbus controller via the terminal bus. The fieldbus controller is connected to the soft-PLC using the Transmission Control Protocol/ Internet Protocol (TCP/IP) Interface. The communication between fieldbus controller and soft-PLC is carried out in the Modbus-Protocol format. The soft-PLC sends out a specific request (Request) in the Modbus-Protocol formal to the fieldbus controller and receives the requested value from the process image (Response).

As soon as the response has been received by the soft-PLC, the requested data are available for the signal processing. Both, ModbusRequest and Modbus-Response, consist of binary codes and are organised as bytes. The runtime platform with soft-PLC is the Modbus-

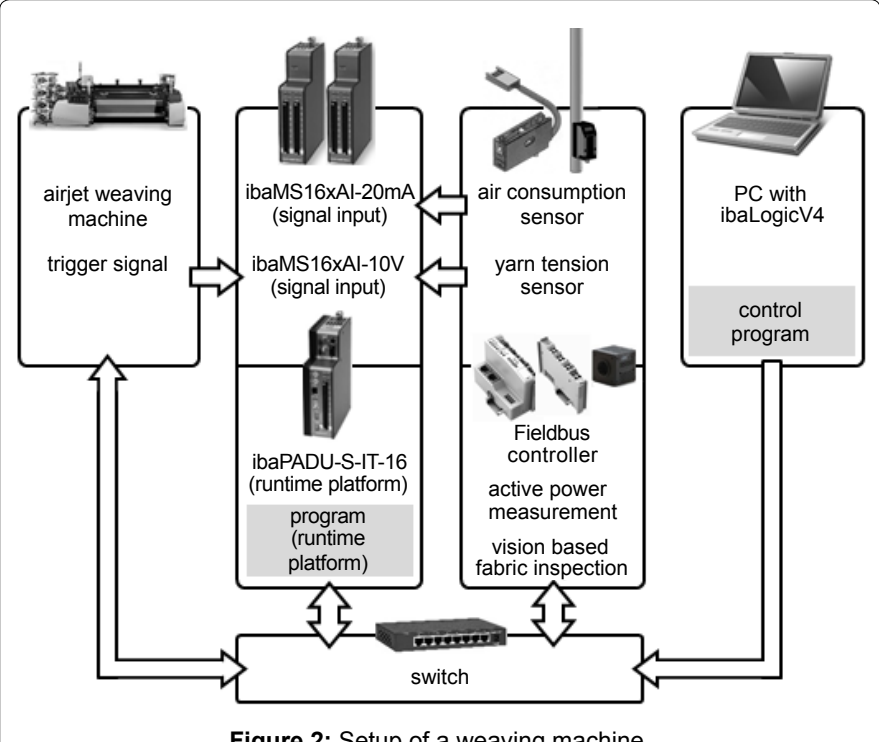

Figure 2: Setup of a weaving machine. 
Master and sends the request to the fieldbus controller, which is the Modbus-Slave. The request contains information regarding the requested value from the process image of the power measurement module. In response to the request the fieldbus controller identifies the requested value and stores it into the response. The response is send via TCP/IP interface to the Modbus-Master. In the scope of this paper the active power is requested from the process image of the power measurement module.

Measurement technology for fabric quality: At Institut für Textiltechnik der RWTH Aachen University (ITA), Aachen, Germany a measuring system for online error detection during fabric production was developed [6]. A camera takes pictures of the fabric. Subsequently the pictures are checked for defects in the fabric, using digital image processing. The software for digital image processing runs on a separate computer. The camera system is installed over the section of the weaving machine where the fabric is created, as shown in Figure 3.

The camera system is able to detect defects immediately after the fabric is produced. The digital image processing software is calibrated using a flawless piece of fabric. The digital image processing classifies deviations from the calibrated condition as a defect [6]. Depending on the share of incorrect pixels in the pictures, the fabric is assigned to a quality category.

The examination for defects is carried out in real time during the weaving process. The computer running the digital image processing is connected to the soft-PLC via TCP/IP interface. Depending on the status of the fabric, the number of the quality category (0 to 4 ) is continuously transmitted via TCP/IP. A quality category of 0 is achieved in case the fabric quality is accurate. A quality category of 4 stands for a destroyed fabric.

\section{Program steps of multi-objective self-optimization}

The program for MOSO consists of the steps shown in Figure 4. Though continuous communication between weaving machine and soft-PLC, the weaving machine is enabled to run the entire program autonomously.

In the first step an experimental design is calculated automatically. Within this design, the three setting parameters static warp tension, vertical position of warp stop motion and revolutions per minute are varied. The user sets the parameter spaces to ensure that the algorithm acts within a feasible range.

During the second step, the test procedure, the weaving machine sets-up every test point. Sensor data describing the objective functions are recorded for the respective parameter setting.

In the third step, the obtained data are used to calculate three regression models (one model per each objective function) which describe the objective functions in dependence of the setting parameters.

In the last step, an optimized set-up of the weaving machine based on predefined quality criteria is calculated by application of desirability functions and a numerical optimization algorithm. Before execution of the optimisation procedure, user-defined preferences regarding the objective functions (warp tension, energy consumption and fabric quality) can be integrated through target weights. The preference scale for each objective function is divided into three sections (low, middle, high).

The program for MOSO is implemented within the ibaLogicV4 programming environment and runs on a central processing unit as depicted in section II.A.

The next chapter illuminates desirability functions and the optimization algorithm used for MOSO.

\section{Desirability Functions and Nelder/Mead Algorithm}

\section{Desirability functions}

The origin of the application of desirability functions in the multidimensional optimization goes back to Derringer and Suich [7]. The aim of using desirability functions is to summarize the objective functions which need to be optimized into one common function. The aggregation of the objective functions is conducted using the so-called desirability. For each objective function, one desirability function is developed. The desirability function assigns a desirability to each value of the objective functions. The desirability function has a value range of $(0 ; 1)$. If the value of one objective function reaches a desirability of zero, the result is invalid within the optimization routine. In case the desirability reaches the value one, the value of the objective function is optimal. In Figure 5 the exemplified shape of a desirability function is shown. The desirability $\mathrm{w}_{\mathrm{Z}}$ is plotted over the normalized objective function $\mathrm{Z}(\mathrm{X})$. Desirability functions can be constructed in three different ways, as shown in Figure 5. If the goal is to achieve the highest possible value for one objective function, the desirability $\mathrm{f}$ unction for aximizing has to be used. The desirability increases when the objective function value increases, etc.

The aim of the utilization of desirability functions is to aggregate the target functions into one common function, the so-called total desirability $\mathrm{d}_{\text {tot }} \cdot \mathrm{d}_{\text {tot }}$ is calculated by using the geometric mean of the individual desirabilities:

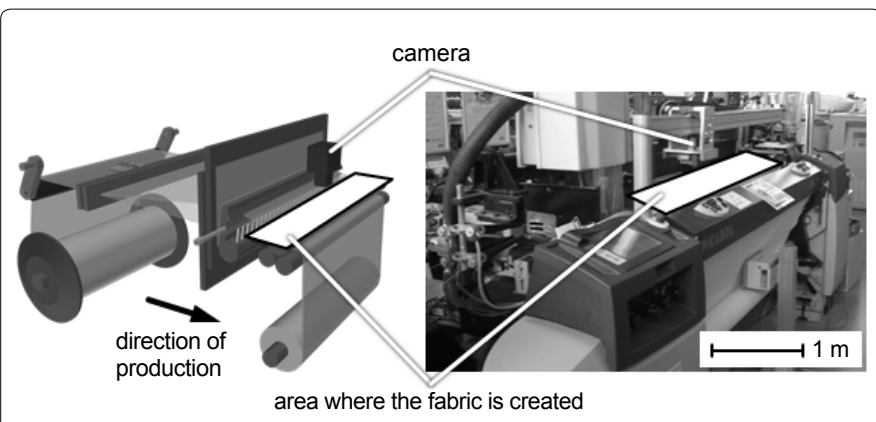

Figure 3: Vision-based defect detection of woven fabrics according to [6]

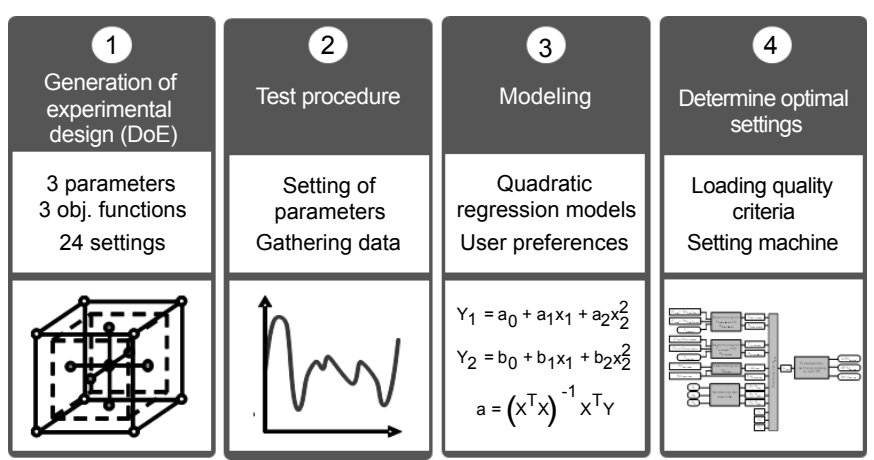

Figure 4: Program steps of Multi-Objective Self-Optimization of the Weaving Process 


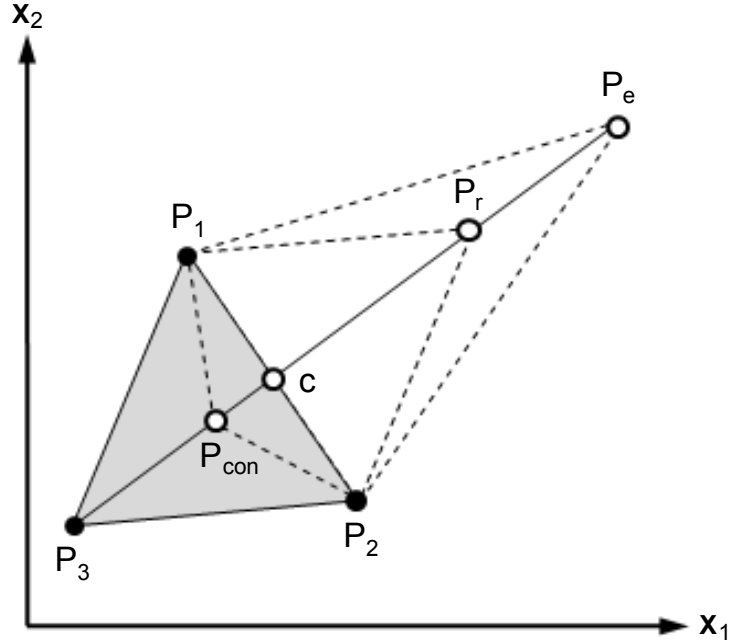

Figure 5: Basic operations of Nelder/Mead algorithm by the example of two parameters $x_{1}$ and $x_{2}\left(P_{1}, P_{2}, P_{3}\right.$ : Calculated objective function values by algorithm; c: focal point; $P_{r}$ : reflection; $P_{e}$ : expansion; $P_{\text {con }}$ : contraction.

$$
\mathrm{d}_{\mathrm{tot}}=\left(\mathrm{w}_{1} \cdot \mathrm{w}_{2} \cdot \ldots \cdot \mathrm{w}_{\mathrm{n}}\right)^{1 / \mathrm{n}}
$$

Whereas $\mathrm{w}_{1}, \mathrm{w}_{2}, \ldots, \mathrm{w}_{\mathrm{n}}$ are the desirabilities of $\mathrm{n}$ objective functions.

The total desirability reveals how close the individual desirabilities are to the optimal range. Because of the multiplication of the individual desirabilities, $d_{\text {tot }}$ is in the range of $(0 ; 1)$. A total desirability of one is reached, when all target functions are in the optimal range. In case only one target function has an invalid value, the total desirability equals to zero.

The combination of process parameters which maximizes $d_{\text {tot' }}$ represents the optimal operating point for the weaving process.

Numeric algorithms are suitable for maximizing the total desirability. The application of numeric algorithms is more efficient than for example grid search methods [8]. It is advised in several references to utilize Nelder/Mead algorithm [9] to maximize the total desirability, see e.g. [10,11].

\section{Nelder/mead algorithm}

The Nelder/Mead algorithm is a numeric optimization procedure [9]. To find a subjective optimal operating point of the weaving machine, $d_{\text {tot }}$ is maximized. The Nelder/Mead algorithm searches for a combination of the three parameters basic warp tension, revolutions per minute and vertical warp stop motion position that maximizes $d_{\text {tot }}$. The basic operations of Nelder/Mead algorithm are shown in Figure 6.

Setting the start values for the considered parameters leads to the starting point for the algorithm. The start values are set before the first iteration and are moved towards the optimal values during the utilization of the algorithm. Starting from a minimization problem with $\mathrm{m}$ parameters, the algorithm considers $\mathrm{m}+1$ parameter combinations $\left(\mathrm{P}_{1}, \mathrm{P}_{2}, \ldots, \mathrm{P}_{\mathrm{m}+1}\right) \mathrm{P}_{1}$. The values of the objective functions functions are calculated in the $m+1$ points and sorted ascendingly. Figure 6 shows the minimization problem for a function of two parameters. The next step is the examination of the three points $\mathrm{P}_{1}, \mathrm{P}_{2}$ and $\mathrm{P}_{3}$ in the parameter space. At each of the three points the algorithm calculates the value ob the objective functions $\mathrm{F}\left(\mathrm{x}_{1}, \mathrm{x}_{2}\right)$ :

$$
\mathrm{F}_{\mathrm{i}}=\mathrm{F}\left(\mathrm{P}_{\mathrm{i}}\right), \mathrm{i}=1,2,3
$$

Afterwards the function values are sorted:

$$
\mathrm{F}_{1} \leq \mathrm{F}_{2} \leq \mathrm{F}_{3}
$$

Considering this example, $\mathrm{F}_{3}$ is the worst (highest) and $\mathrm{F}_{1}$ is the best (lowest) value in the context of the optimization. The minimization of the target function is achieved by applying several iterations of the algorithm. In each iteration one new point in the parameter space is created, which replaces the point $\mathrm{P}_{\mathrm{m}+1}$ with the biggest value of the function to be minimized. In the present case the point $\mathrm{P} 3$ results is the worst value of the target function and is therefore replaced in the next iteration. The replacement of the worst point is achieved through the basic operations of the Nelder/Mead algorithm which are visualized in Figure 6.

\section{Experimental Results}

In this chapter, the ibaLogicV4 program for MOSO of the weaving process is validated during a long-term test in the laboratory of ITA. To establish industrial conditions, the duration of the long-term test is eight hours, like usual shift duration. A long-term test is carried out using the MOSO against not using the optimization procedure respectively, to examine the influence of $\mathrm{MOSO}$ on production figures. The long-term test is conducted with an air-jet weaving machine OmniPlus 800 by Picanol n.v., Ieper, Belgium. During the long-term test, polyester filament yarn with 330 dtex for warp and weft was used (binding: twill 3/1). The configuration of MOSO used for the long-term test are listed in Table 1.

After program execution of MOSO (Figure 4), the algorithm calculates the following optimal parameter settings: $b w t=3,71 \mathrm{kN}$; $\mathrm{n}=522$ RPM; $\mathrm{wsm}_{\mathrm{y}}=20 \mathrm{~mm}$.

The following settings are used as reference settings coming from an
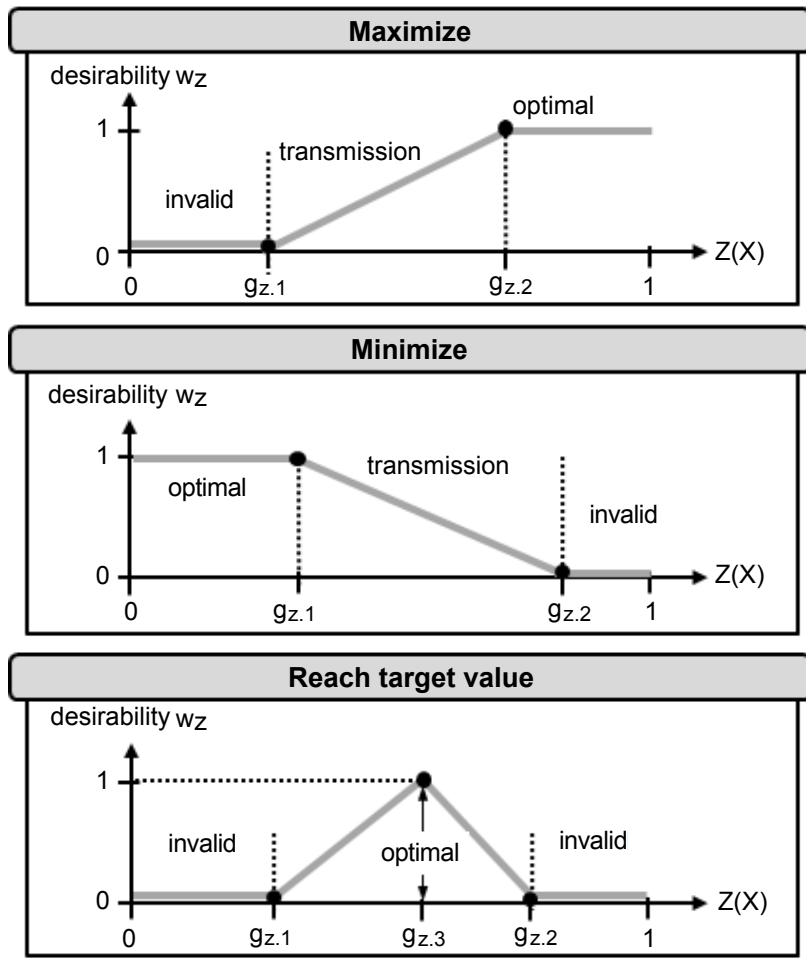

Figure 6: Examplary layout of desirability functions [7] 
Citation: Saggiomo M, Gloy YS, Gries T (2016) Reduction of the Weaving Process Set-up Time through Multi-Objective Self-Optimization. J Textile Sci Eng 6: 255. doi:10.4172/2165-8064.1000255

Page 5 of 6

\begin{tabular}{|c|c|}
\hline Setting & Value \\
\hline Lower/upper limit bwt & $2 \mathrm{kN} / 4 \mathrm{kN}$ \\
\hline Lower/upper limit $\mathrm{n}$ & $400 \mathrm{RPM} / 900 \mathrm{RPM}$ \\
\hline Lower/upper limit wsm & $\mathrm{y}$ \\
\hline Target weight warp tension & LOW \\
\hline Target weight energy consumption & LOW \\
\hline Target weight quality & HIGH \\
\hline Algorithm start point bwt/n/wsm & $\mathrm{mm}$ \\
\hline
\end{tabular}

Table 1: Configuration of MOSO used for long-term test.

\begin{tabular}{|l|c|c|}
\hline \multirow{2}{*}{ Recorded data } & \multicolumn{2}{|c|}{ Results } \\
\cline { 2 - 3 } & MOSO & Ref. settings \\
\hline Efficiency (prod. time/total time) & $98,6 \%$ & $97,2 \%$ \\
\hline Produced fabric & $8,16 \mathrm{~m}$ & $15,41 \mathrm{~m}$ \\
\hline Weft insertions & 125157 & 215982 \\
\hline Weft defects & 2 & 6 \\
\hline Warp breakages & 0 & 0 \\
\hline Average warp tension & $1,27 \mathrm{~N}$ & $1,49 \mathrm{~N}$ \\
\hline Average air consumption & $134,23 \mathrm{~m}^{3} / \mathrm{h} \mathrm{i.} \mathrm{N}$. & $155,26 \mathrm{~m}^{3} / \mathrm{h} \mathrm{i.} \mathrm{N}$. \\
\hline Average active power usage & $2,49 \mathrm{~kW}$ & $4,62 \mathrm{~kW}$ \\
\hline Average quality category & 0,93 & 1,55 \\
\hline Set-up time & $30 \mathrm{Min}$. & $120 \mathrm{Min}$. \\
\hline
\end{tabular}

Table 2: Results of long-term test.

industrial weaving mill that processes the same material as mentioned above: bwt $=4 \mathrm{kN} ; \mathrm{n}=900 \mathrm{RPM} ; \mathrm{wsm}_{\mathrm{y}}=0 \mathrm{~mm}$. During the long-term test the following parameters of the weaving process are recorded:

- efficiency of the weaving machine

- produced amount of fabric

- amount of weft insertions

- warp/weft defects and breakages

Additionally, data of the objective functions are recorded. The results of the long-term test using MOSO and reference settings are shown in Table 2 .

During the long-term test, sensor data regarding the objective functions are recorded using the software ibaPDA from iba AG, Fürth, Germany. The measured data is illustrated in Figures 7-10. Data are plotted over the main shaft position of the weaving machine which is the rotating angle of the machine's main drive.

The program for self-optimization enables the weaving machine to autonomously find an operating point, which improves all objective functions compared to conventional (reference) machine settings.

The target weight of fabric quality was set high and correlates with the course of the quality category displayed in Figure 10. MOSO is able to find an operating point of the weaving machine, in which the quality category reaches the optimal value of zero almost constantly.

The efficiency presents the relation of production time of the weaving machine to the total time. The efficiency of the weaving machine is higher using the optimal setting than in case of using the reference settings, see Table 2 . Higher efficiency is mainly achieved by reduced machine downtime.

Using the optimal machine settings, two weft defects caused be the collision of the weft threads with sagging warp threads occurred.
In contrast, using the suboptimal settings, six weft defects occurred. During the long-term test it was observed, that the machine runs more stable with less RPM. The higher amount of weft defects can be explained by a disadvantageous machine speed of 900 RPM. Weft defects result from the faulty transport of weft threads across the width of the weaving machine.

Without MOSO a machine operator needs around $120 \mathrm{~min}$. for the configuration of the weaving machine and to find appropriate settings for the process. The program for self-optimization is concluded in 30 minutes and successfully reduces the set-up time by $75 \%$.

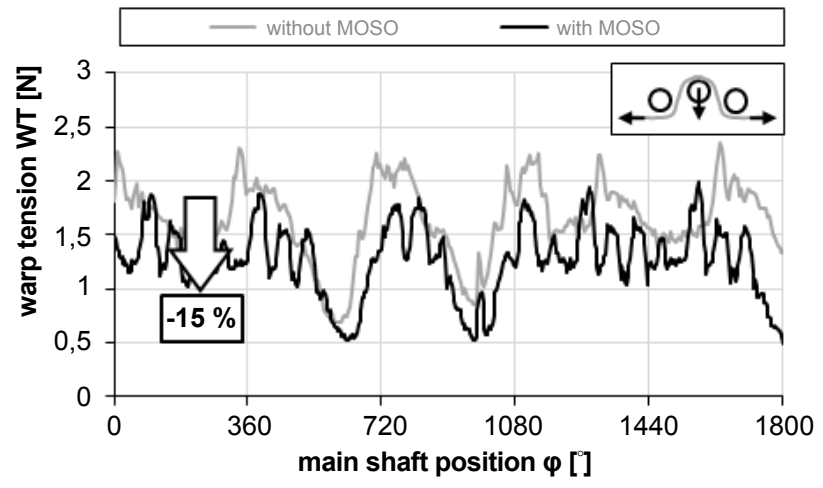

Figure 7: Comparison of warp tension with and without MOSO.

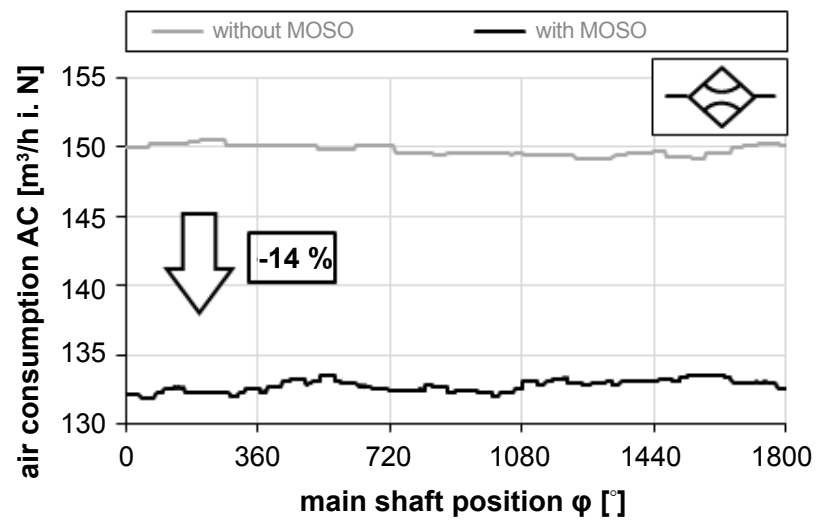

Figure 8: Comparison of air consumption with and without MOSO.

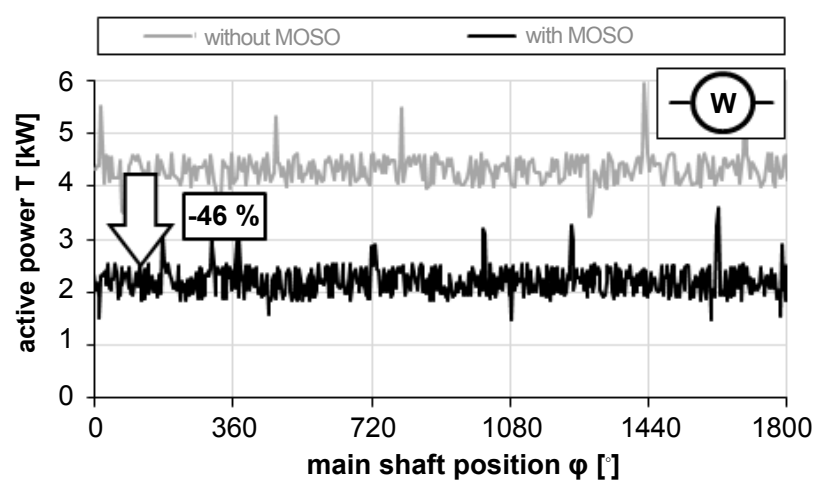

Figure 9: Comparison of active power consumption with and without MOSO 
Citation: Saggiomo M, Gloy YS, Gries T (2016) Reduction of the Weaving Process Set-up Time through Multi-Objective Self-Optimization. J Textile Sci Eng 6: 255. doi:10.4172/2165-8064.1000255

Page 6 of 6

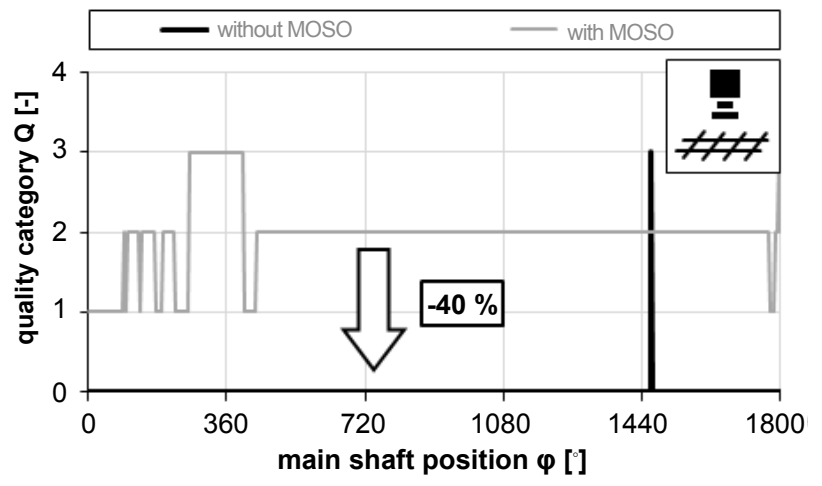

Figure 10: Comparison of fabric quality with and without MOSO.

\section{Summary and Outlook}

This paper presented a concept and implementation of multiobjective self-optimization of the weaving process. An optimization routine which is implemented into a software-based programmable logic controller enables the weaving machine to calculate optimal parameter settings autonomously. Individual preferences of operators or plant management are integrated into the calculations. By assistance of the resulting cognitive weaving machine, the set-up time was reduced by $75 \%$.

Further research will focus the development of mobile applications to form an assistance system for operators basing on the presented results. Moreover, the cognitive weaving machine will be embedded into an intelligent textile process chain in the sense of Industrie 4.0 where all production units are interconnected to each other.

\section{Acknowledgments}

The authors would like to thank the German Research Foundation DFG for the kind support within the Cluster of Excellence "Integrative Production Technology for High-Wage Countries".

\section{References}

1. Brecher C (2011) Integrative Production Technology for High-Wage Countries. Springer-Verlag, Berlin/Heidelberg 1: 747-1057.

2. Osthus T (1996) Process optimization and changeover time reduction for weaving through automatical adjustment of backrest and warp stop motion. Dissertation, Rheinisch Westfälische Technische Hochschule Aachen, Aachen, Germany. Original title: "Prozessoptimierung und Rüstzeitverkürzung in der Weberei durch automatische Einstellung von Streichbaum und Kettwächterkorb“

3. Chen M (1996) Computergestützte Optimierung des Webprozesses bezüglich Kettfadenbeanspruchung und Kettlaufverhalten. Dissertation, Universität Stuttgart, Stuttgart, Germany.

4. Adanur S (2001) Handbook of Weaving. CRC press, Taylor and Francis, Boca Raton, London, New York.

5. Gausemeier J, Rammig FJ, Schäfer W, Josef F (2009) Design Methodology for Intelligent Technical Systems: Develop Intelligent Technical Systems of the Future. Springer Science and Business Media.

6. Schneider D, Gloy YS, Merhof D (2015) Vision-Based On-Loom Measurement of Yarn Densities in Woven Fabrics. Instrumentation and Measurement, IEEE Transactions 64: 1063-1074.

7. Derringer G, Suich R (1980) Simultaneous Optimization of Several Response Variables. Journal of Quality Technology 4: 214-219.

8. Blobel V, Lohrmann E (1998) Statistische und numerische Methoden der Datenanalyse.

9. Nelder JA, Mead R (1965) A Simplex Method for Function Minimization. The Computer Journal 4: 308-313.

10. Bera S, Mukherjee I (2010) Performance Analysis of Nelder-Mead and A Hybrid Simulated Annealing for Multiple Response Quality Characteristic Optimization. Proceedings of the International MultiConference 3: 1728-1732

11. Gloy YS, Sandjaja F, Gries T (2015) Model-based self-optimization of the weaving process. Journal of Manufacturing Science and Technology (CIRP) 9: 88-96. 